\title{
Novel Solution for Multi-connectivity 5G-mmW Positioning
}

\author{
(This is a preprint of an accepted article scheduled to be presented at \\ The Asilomar Conference on Signals, Systems, and Computers, to be held October 28-31, 2018, \\ in Asilomar Grounds in Pacific Grove, CA, USA.) \\ Jani Saloranta*, Giuseppe Destino*†, Antti Tölli*, Henk Wymeersch ${ }^{\ddagger}$ \\ *University of Oulu, Oulu, Finland \\ ${ }^{\dagger}$ King's College London, London, United Kingdom \\ $\ddagger$ Chalmers University of Technology, Gothenburg, Sweden \\ Email: \{jani.saloranta,giuseppe.destino,antti.tolli\}@oulu.fi, henkw@chalmers.se
}

\begin{abstract}
The forthcoming fifth generation (5G) systems with high beamforming gain antenna units, millimeter-wave (mmWave) frequency bands together with massive Multiple Input Multiple Output (MIMO) techniques are key components for accurate positioning methods. In this paper, we propose the positioning technique that is relying on the sparsity in the MIMO-OFDM channel in time and spatial domains, together with effective beamforming methods. We will study the proposed solution in a multi-connectivity context, which has been considered so far for the purpose of improving the user equipment (UE) communication data rate. We utilize the multi-connectivity for positioning, in order to improve robustness to measurement errors and increase positioning service continuity. In particular, we show that when a UE that has connectivity to more base stations, the total power and delay needed for positioning can be reduced.
\end{abstract}

\section{INTRODUCTION}

$5 \mathrm{G}$ millimeter-wave (mmWave) signals are characterized by large bandwidths and will be sent and received over large arrays. Combined, this leads to high potential for distance and angle estimation [1]. Applications include vehicular positioning [2] and location-aided communications [3]. An important benefit of mmWave is the reduced requirements on anchor deployment, as localization is possible with only few [4] or even a single anchor [5].

A property of mmWave communication is the use of few radio frequency (RF) chains, relying on analog or hybrid precoders and combiners to establish a high-SNR link [6]. This implies that mmWave localization must account for this hardware limitation in the design of signals and algorithms. For instance, [7] derived the optimal beamforming solution for a given scenario that minimizes the Cramér Rao lower bound (CRLB) for a multicarrier mmWave system in terms of the CRLB characterizing angle-of-arrival (AoA) and delay estimation. In order to design signals to can cover a large angular uncertainty, [8] proposed a 5G mmWave user positioning method by means of beamformed downlink reference signals, while [9] formulated an optimization problem to optimize power allocation across subcarriers for a fixed a priori position uncertainty of the user, given a certain a posteriori requirement on the user uncertainty.

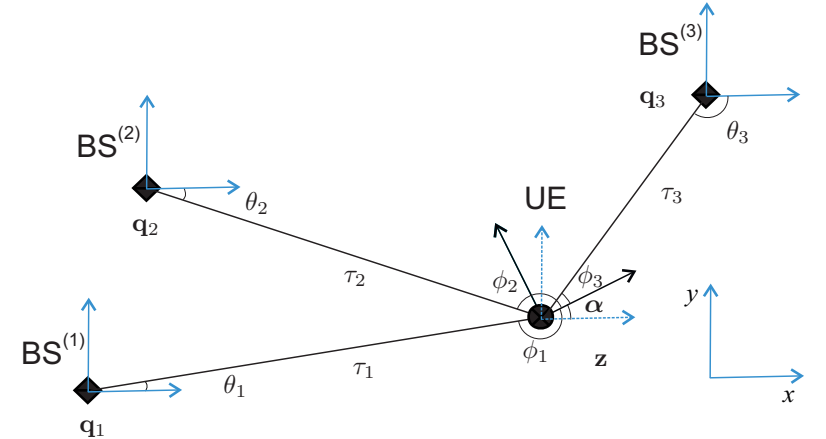

Fig. 1. Communication model showing 3 transmitters $(\mathrm{gNB})$ at location $\mathbf{q}_{\mathbf{i}}$ and receiver (UE) at location $\mathbf{z}$ with LOS delays denoted by $\tau_{i}$ and angles AoA $\phi_{i}$, AoD $\theta_{i}$, and the rotation $\alpha$ of the receiver.

In this paper, we employ a similar formulation as [9] and [7], whereby we aim to find the optimal power allocation for different beam pairs in a multi base station (gNB) scenario. In particular, we are interested in gaining understanding in (i) to what extent it is beneficial to use more than one base station; (ii) how does the total allocated power vary based on the localization requirement, optimization criterion, and number of base stations; (iii) how do power and delay trade off when using more than one base station?

\section{SySTEM MOdEL}

We consider a two-dimensional scenario with a single user (UE) and $N_{b s}$ base stations (gNBs). The user's position and orientation $^{1}$, respectively denoted by $\mathbf{z} \in \mathbb{R}^{2}$ and $\alpha$, are unknown and yet to be determined. Furthermore, each gNB as well as the user have multiple antennas for beamforming and for simplicity, a single radio frequency (RF) chain transceiver architecture is considered. More specifically, we denote the $b$-th gNB's location $\mathbf{q}_{b}$ and we shall assume that all $\mathrm{gNBs}$ ' antenna comprises $N$ elements, whereas UE's antenna has $M$ elements.

\footnotetext{
${ }^{1}$ The orientation of the user is measured with respect to a global reference system.
} 
At the time-slot $n$, the received signal at the $\mathrm{UE}$ is given by the superposition of $N_{b s}$ orthogonal reference signals, i.e.,

$$
\begin{aligned}
& y_{n}(t)=\sum_{b=1}^{N_{b s}} \sqrt{p_{n b}} h_{b} \mathbf{w}_{n b}^{\mathrm{H}} \mathbf{a}_{M}\left(\theta_{b}\right) \mathbf{a}_{N}^{\mathrm{H}}\left(\phi_{b}\right) \mathbf{f}_{n b} x_{b}\left(t-\tau_{b}\right) \\
& +\mathbf{w}_{n b}^{\mathrm{H}} \mathbf{n}_{n b}(t),
\end{aligned}
$$

where $h_{b} \in \mathbb{C}, \tau_{b} \phi_{b}$ and $\theta_{b}$ are the complex channel gain, path-delay, AoA and angle-of-departure (AoD) of the line-of-sight (LOS) link between the $b$-th gNB and the UE, $\mathbf{f}_{n b} \in \mathcal{C}_{b}$ and $\mathbf{w}_{n b} \in \mathcal{C}_{u}$ are the transmit-receive unitnorm beamforming pair used for $(n, b)$-th reference signal transmission; $\mathcal{C}_{b} \subset \mathbb{C}^{N}$ and $\mathcal{C}_{u} \subset \mathbb{C}^{M}$ are the codebook of the $b$-th gNB and UE, respectively. In addition, $\mathbf{n}_{n b}(t)$ is the Additive White Gaussian Noise (AWGN) with power spectral density (PSD) $N_{0}, x_{b}(t)$ is the reference signal transmitted by the $b$-th $\mathrm{gNB}$ with bandwidth $B$, duration $T_{\mathrm{sym}}$ and unit power, i.e., $1 / T_{\text {sym }} \int_{0}^{T_{\mathrm{sym}}}|x(t)|^{2} \mathrm{~d} t=1$. Finally, we denote by $\mathbf{a}_{N}(x) \in \mathbb{C}^{N}$ and $\mathbf{a}_{M}(x) \in \mathbb{C}^{M}$, the transmit and receive array response vectors of the gNB $b$ and UE, respectively.

Positioning is performed by the joint processing of $N_{p}$ transmissions, i.e., based on the receiving vector $\mathbf{y}(t) \in \mathbb{C}^{N_{p}}$. The objective of this work is to develop an power-allocation strategy that minimizes the total transmit power (over all beam pairs and all gNBs) subject to a constraint of the user localization uncertainty. More specifically, our optimization problem is defined as follows:

$$
\begin{array}{ll}
\min _{\mathbf{P}} & \mathcal{P}(\mathbf{P}) \\
\text { s.t. } & \text { positioning constraint } \\
& \mathbf{p}_{b} \succeq \mathbf{0} \forall b,
\end{array}
$$

where $\mathcal{P}$ is a power allocation cost function, $\mathbf{P} \triangleq$ $\left[\mathbf{p}_{1}, \cdots, \mathbf{p}_{N_{b s}}\right], \mathbf{p}_{b} \in \mathbb{R}^{N_{p}}$ is the beam-power allocation vector at the $b$-th $\mathrm{gNB}$, and the positioning constraint ensures that good quality of positioning. This quality will be described through the Fisher information and the Cramér-Rao Lower Bound (CRLB), described next.

\section{OPTIMIZATION FORMULATION}

\section{A. FIM Definitions}

In this section, we briefly describe the main FIM concepts. The FIM of $\boldsymbol{\eta}_{b} \triangleq\left[\tau_{b}, \theta_{b}, \phi_{b}, \Re\left(h_{b}\right), \Im\left(h_{b}\right)\right]$ is given by $\mathbf{J}\left(\boldsymbol{\eta}_{b}\right) \in \mathbb{R}^{5 \times 5}$ and the FIM of $\tilde{\boldsymbol{\eta}}_{b} \triangleq\left[\mathbf{z}^{\mathrm{T}}, \alpha, \Re\left(h_{b}\right), \Im\left(h_{b}\right)\right]$ as $\mathbf{J}\left(\tilde{\boldsymbol{\eta}}_{b}\right) \in \mathbb{R}^{5 \times 5}$ [5]. These are related by $\mathbf{J}\left(\tilde{\boldsymbol{\eta}}_{b}\right)=\mathbf{T}_{b}^{\mathrm{T}} \mathbf{J}\left(\boldsymbol{\eta}_{b}\right) \mathbf{T}_{b}$, where $\mathbf{T}_{b} \in \mathbf{R}^{5 \times 5}$ is $\mathbf{T}_{b} \triangleq\left(\partial \boldsymbol{\eta}_{b}^{T}\right) /\left(\partial \tilde{\boldsymbol{\eta}}_{b}\right)$. The Equivalent Fisher Information Matrix (EFIM) $\mathbf{J}_{e, b}(\mathbf{z}) \in \mathbb{R}^{2 \times 2}$ is defined as the inverse of the first $2 \times 2$ block of $\mathbf{J}_{\tilde{\boldsymbol{\eta}}_{b}}^{-1}$. The information from different $\mathrm{gNBs}$ is additive so that the total EFIM is

$$
\mathbf{J}_{e}(\mathbf{z})=\sum_{b=1}^{N_{b s}} \mathbf{J}_{e, b}(\mathbf{z}) .
$$

Additional information on the expressions of the FIM and EFIM can be found in [5], [10]. The inverse of the EFIM serves as a lower bound on the localization error covariance.
Remark 1. Note that FIMs $\mathbf{J}\left(\tilde{\boldsymbol{\eta}}_{b}\right)$ cannot be added since are defined over different parameters (individual $h_{b}$ ). Instead one can define a FIM over $\tilde{\boldsymbol{\eta}} \triangleq\left[\mathbf{z}^{\mathrm{T}}, \alpha,\left[\Re\left(h_{b}\right), \Im\left(h_{b}\right)\right]_{b=1}^{N_{b s}}\right] \in \mathbb{R}^{3+2 N_{b s}}$.

\section{B. FIM-constrained Optimization Problems}

Given a selection of beams $\mathbf{f}_{n b}$ from gNB $b$ and corresponding combiners $\mathbf{w}_{n b}$, then the FIM and EFIM are additive, i.e.,

$$
\mathbf{J}_{e, b}(\mathbf{z})=\sum_{\substack{\mathbf{w}_{n b} \in \mathcal{C}_{u} \\ \mathbf{f}_{n b} \in \mathcal{C}_{b}}} p_{b n} \mathbf{J}_{e, b}\left(\mathbf{w}_{n b}, \mathbf{f}_{n b} ; \mathbf{z}\right),
$$

in which we have explicitly extracted the power $p_{b n}$, so that $\mathbf{J}_{e, b}\left(\mathbf{w}_{n b}, \mathbf{f}_{n b} ; \mathbf{z}\right)$ should be interpreted as the EFIM of $\mathbf{z}$ given $\mathbf{w}_{n b}, \mathbf{f}_{n b}$ and unit power.

A meaningful optimization is then

$$
\begin{array}{ll}
\min _{\mathbf{p}} & \|\mathbf{p}\|_{\alpha} \\
\text { s.t. } & \mathbf{J}_{e}^{-1}(\mathbf{z}) \preceq \frac{\gamma_{e}}{2} \mathbf{I}_{2}, \\
& \mathbf{p} \succeq \mathbf{0},
\end{array}
$$

where $\|\cdot\|_{\alpha}$ denotes a $\ell_{\alpha}$ matrix norm, $\mathbf{p} \in \mathbb{R}^{N_{b s} N_{p}}$ is the vectorized form of $\mathbf{P}$ and $\alpha$ can be $\{1,2, \infty\}$. More specifically, $\alpha=1$ is used to seek the minimum number of beams, $\alpha=2$ is used to minimize the average power and $\alpha=\infty$ is used to minimize a constant power. Moreover, $\mathbf{J}_{e}(\mathbf{z})$ is given by (3) and (4). The constraint $\mathbf{J}_{e}^{-1}(\mathbf{z}) \preceq \gamma_{e} / 2 \mathbf{I}_{2}$ ensures that the location error variance is less than $\gamma_{e} / 2$ in each dimension. We note that the widely used metric Position Error Bound (PEB) is given by $\sqrt{\gamma_{e}}$. We can express the optimization problem equivalently using a positive semidefinite matrix constraint as

$$
\begin{array}{ll}
\min _{\mathbf{p}} & \|\mathbf{p}\|_{\alpha} \\
\text { s.t. } & {\left[\begin{array}{cc}
\frac{\gamma_{e}}{2} \mathbf{I}_{2} & \mathbf{I}_{2} \\
\mathbf{I}_{2} & \mathbf{J}_{e}(\mathbf{z})
\end{array}\right] \succeq 0 .} \\
& \mathbf{p} \succeq \mathbf{0},
\end{array}
$$

which is a convex optimization problem and thus amenable for efficient numerical solving. Note that additional constraints (e.g., a power constraint per gNB are straightforward to include).

Remark 2. An alternative optimization can be formulated by determining the FIM of $\tilde{\boldsymbol{\eta}}$ and enforcing $\mathbf{J}^{-1}(\tilde{\boldsymbol{\eta}}) \preceq \gamma_{e}$, where $\gamma_{e} \in \mathbb{R}^{3+2 N_{b s}}$ is a diagonal matrix comprising the limits on the error variance of each component. The same approach can then be used.

\section{Simulation Results}

In this section, we consider a single-user multi-base station scenario and compare three power allocation strategies, hereafter referred to as: 1$)$ minimun number of beams $\left(\ell_{1}\right.$-norm based), 2) minimum average power ( $\ell_{2}$-norm based) and 3 ) minimum constant power ( $\ell_{\infty}$-norm based). 


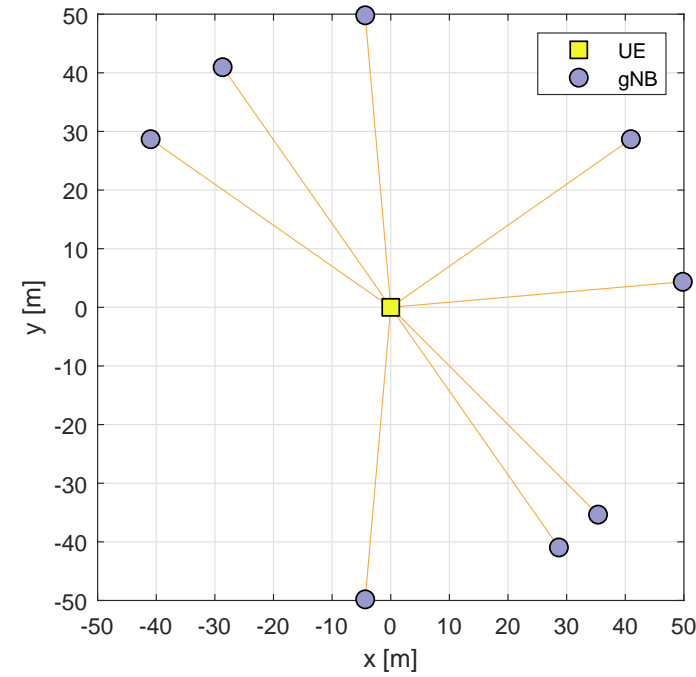

Fig. 2. Illustration of simulation scenario with 8 randomly placed gNBs around the UE. Distance from each gNB to UE is constant $d_{b}=\left\|\mathbf{z}-\mathbf{q}_{b}\right\|=$ $50 \mathrm{~m} \forall b$, and AoA $\theta_{b} \sim \mathcal{U}(0,2 \pi) \forall b$.

\section{A. Simulation Scenario}

In all simulation scenarios, UE's coordinate vector is $\mathbf{z}=$ $[0,0]^{\mathrm{T}}$ and $N_{b s}$ gNBs are placed randomly on a circle of radius $d$ centred at the UE. For instance, in Fig. 2, a typical scenario with $N_{b s}=8$ and $d=50 \mathrm{~m}$ is illustrated. We assume a downlink orthogonal frequency-division multiplexing (OFDM) communication at $28 \mathrm{GHz}$ with subcarrier spacing $\Delta_{f}=120 \mathrm{kHz}$ and 127 subcarriers. Both UE and gNB have uniform linear array (ULA) antennas, respectively, with 8 and 16 elements. Beamforming is generated in the RF domain, and for simplicity, we consider a single RF chain and Discrete Fourier Transform (DFT) type codebook.

Positioning measurements (angle and ranging) are obtained from a sequential beam scanning, which takes at most $128^{2}$ slots $^{3}$, but might be lower based on the selected powerallocation strategy.

Finally, we shall consider the following metrics: $i$ ) the average total transmit power per gNB to look into overall power consumption of the positioning measurement process, ii) the average number of beams to evaluate the time-efficiency of the measurement process and, iii) the achievable PEB to quantify the effectiveness of power allocation strategy to achieve a target localization accuracy.

\section{B. Results and Discussion}

Fig. 3 shows the average power per gNB in the network to achieve a target accuracy $\sqrt{\gamma_{e}}=0.025 \mathrm{~m}$. The average power means total power over the full setup, i.e., all beam-pairs in the configuration, divided equally per gNB. Generally, with all strategies the average power decreases with the increase of the number of gNBs. In other words, if the optimal localization

\footnotetext{
${ }^{2}$ With the codebook size of UE is $\left|\mathcal{C}_{u}\right|=8$, and each gNB has $\left|\mathcal{C}_{b}\right|=16$.

${ }^{3}$ We assume that in one slot UE can simultaneously receive DL signals from multiple gNBs and, per gNB, only one beam-pair can tested.
}

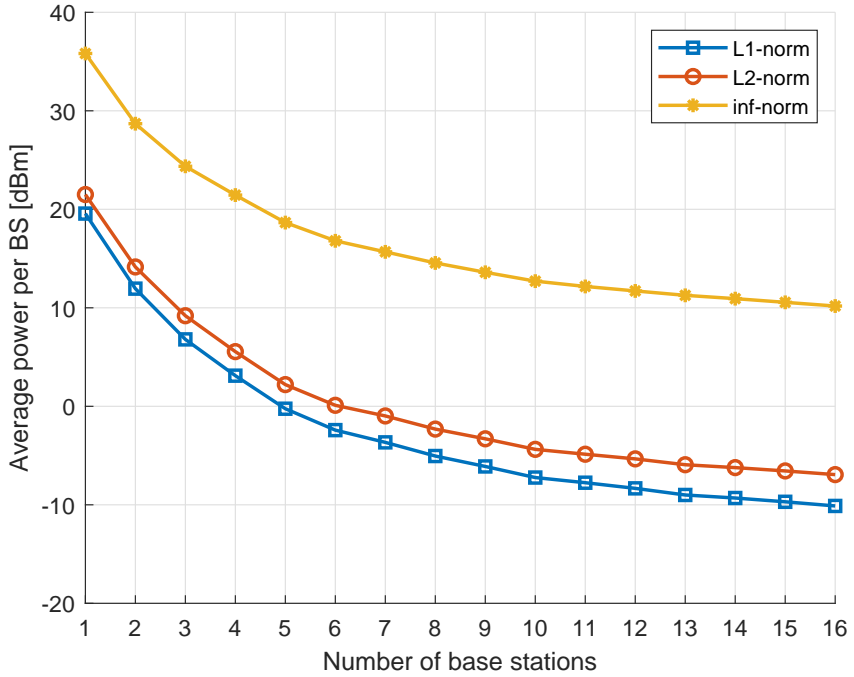

Fig. 3. Required total transmission power over all gNBs and all beam pairs to achieve target PEB $=2.5 \mathrm{~cm}$, for $\ell_{1}, \ell_{2}$ and $\ell_{\infty}$-norms. Average power is total power over all gNBs and beam pairs divided by number of gNBs available in scenario.

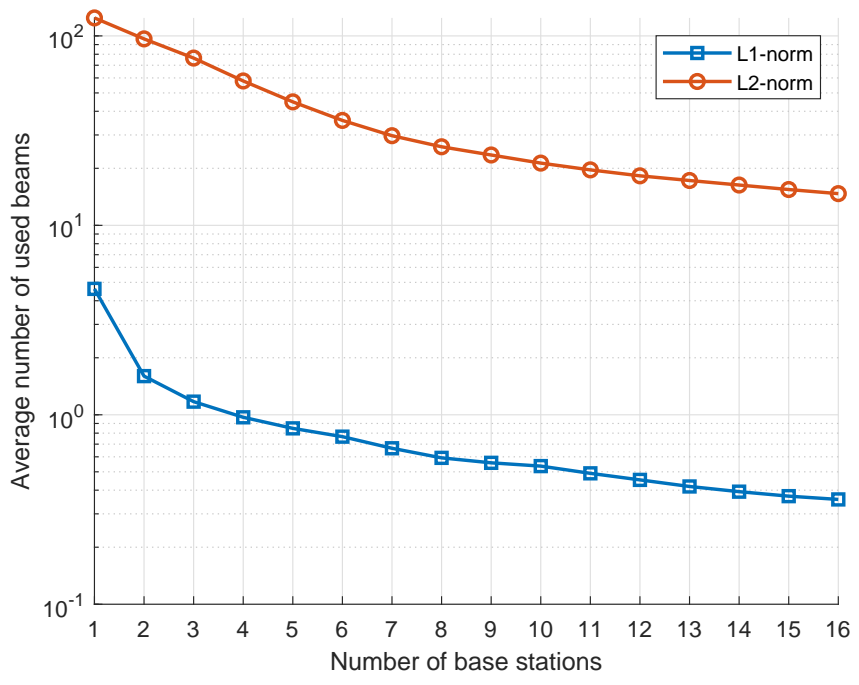

Fig. 4. Average number of used beam pairs (per gNB) as function of number of gNBs in the scenario. ULA antennas used in simulation are $8 \times 1$ and $16 \times 1$ for $\mathrm{UE}$ and for each $\mathrm{gNB}$, respectively.

accuracy is desired, then the most energy-effective solution is to use as many gNB as possible. Consequently, this indicates that using more links for positioning is more energy-effective than increasing the power per beam.

Moreover, by concentrating the power in a few number of beams, strategy 1), yields the lowest power consumption, closely followed by strategy 2). Strategy 3) is the most inefficient solution in terms of power usage.

Fig. 4 shows the average number of used beam pairs - a metric directly related to the measurement time - as a function of the number of gNBs. In this counting, only beam pairs with a transmit power larger than a given threshold are considered, e.g. threshold set to $-80 \mathrm{dBm}$. It can be noticed, that $\ell_{1}$ - 


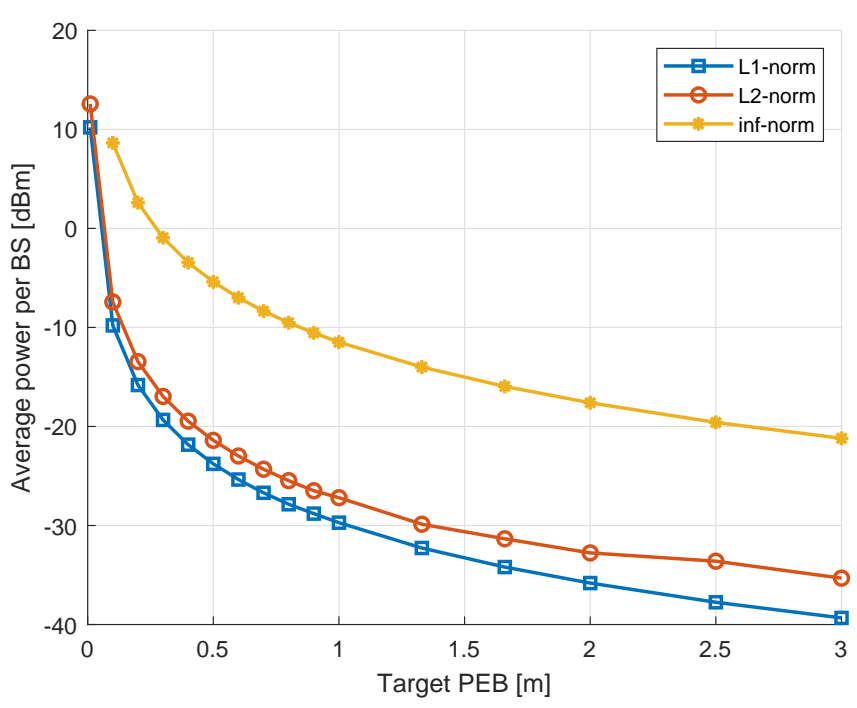

Fig. 5. Total power over gNBs and used beam pairs as function of target PEB. Number of gNBs $N_{b s}=4$.

norm optimization is again the most effective solution as it yields the shortest delay. When $N_{b s}$ increases, the total delay is reduced, even for the $\ell_{2}$ norm. This can be explained by the limited angular resolution of the codebook, so that few beams from more gNBs is better than many beams from few gNBs. For the $\ell_{\infty}$-norm, results are not shown in Fig. 4 as the system always allocates the maximum number of beams.

In Fig. 5, we look into the average total power as a function of target PEB. Generally, a lower target positioning uncertainty leads to a higher power, with a sharp increase for very low values. Finally, in Fig. 6, we consider the realized PEB with respect to the target $\mathrm{PEB}$. Interestingly, the $\ell_{1}$ case leads to a worse PEB than the target, the $\ell_{2}$ case to a PEB close to the target, and the $\ell_{\infty}$ to a PEB smaller than the target. These inconsistencies are due to a mismatch between the channels assumed by the optimizer and those generated after the power was allocated.

\section{CONCLUSION}

In this paper we considered the problem of finding the optimal power allocation over the multiple beams and multiple base stations on MIMO OFDM wireless network. In particular, we formulate a convex optimization problem to allocate power to beam pairs across different base stations. Simulation results indicate that when more base stations are available and there is an overall power constraint, both total power and delay are reduced. In addition, when using and $\ell_{1}$ norm power minimization, the lowest delay with smallest total power are achieved, while $\ell_{2}$-norm power minimization incurs significantly longer delays.

\section{ACKNOWLEDGMENTS}

The research leading to these results has received funding from the Academy of Finland projects 6Genesis Flagship

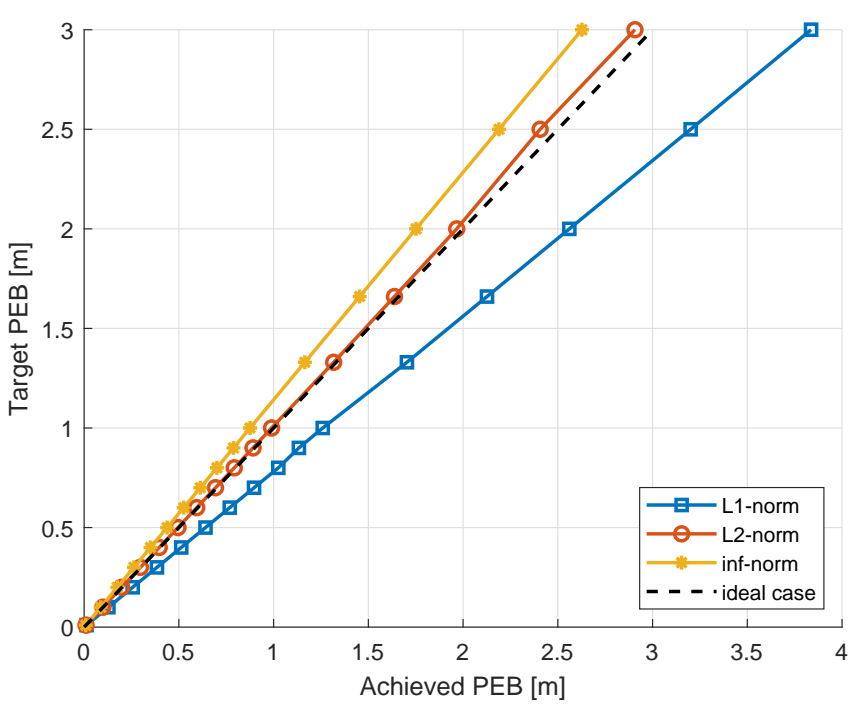

Fig. 6. The performance of the different optimization object functions as a metric of achieved PEB with given target PEB. Black dashed line shows an ideal case. Number of gNBs $N_{b s}=4$.

(Grant No. 318927), Fundamental of Simultaneous Localization and Communications (FALCON), and Positioning-aided Reliably-connected Industrial Systems with Mobile mmWave Access (PRISMA), by the EU H2020 project 5GCAR, and the VINNOVA COPPLAR project, funded under Strategic Vehicle Research and Innovation (Grant No. 2015-04849).

\section{REFERENCES}

[1] R. M. Buehrer, H. Wymeersch, and R. M. Vaghefi, "Collaborative Sensor Network Localization: Algorithms and Practical Issues," Proceedings of the IEEE, vol. 106, no. 6, pp. 1089-1114, Jun. 2018.

[2] H. Wymeersch, G. Seco-Granados, G. Destino, D. Dardari, and F. Tufvesson, "5G mmWave Positioning for Vehicular Networks," IEEE Wireless Commun. Mag., vol. 24, no. 6, pp. 80-86, Dec. 2017.

[3] E. S. Lohan, M. Koivisto, O. Galinina, S. Andreev, A. Tolli, G. Destino, M. Costa, K. Leppanen, Y. Koucheryavy, and M. Valkama, "Benefits of Positioning-Aided Communication Technology in High-Frequency Industrial IoT,' IEEE Commun. Mag., pp. 1-7, 2018.

[4] F. Lemic, J. Martin, C. Yarp, D. Chan, V. Handziski, R. Brodersen, G. Fettweis, A. Wolisz, and J. Wawrzynek, "Localization as a Feature of mmWave Communication," in 2016 International Wireless Communications and Mobile Computing Conference (IWCMC), Sep. 2016, pp. 1033-1038.

[5] A. Shahmansoori, G. E. Garcia, G. Destino, G. Seco-Granados, and H. Wymeersch, "Position and Orientation Estimation Through Millimeter-Wave MIMO in 5G Systems," IEEE Trans. Wireless Commun., vol. 17, no. 3, pp. 1822-1835, Mar. 2018.

[6] S. Kutty and D. Sen, "Beamforming for Millimeter Wave Communications: An Inclusive Survey," IEEE Commun. Surveys Tuts., vol. 18, no. 2, pp. 949-973, 2016.

[7] R. Koirala, B. Denis, D. Dardari, and B. Uguen, "Localization bound based beamforming optimization for multicarrier mmWave MIMO," in 14th Workshop on Positioning, Navigation and Communications (WPNC), Oct. 2017, pp. 1-6.

[8] E. Rastorgueva-Foi, M. Koivisto, M. Valkama, M. Costa, and K. Leppänen, "Localization and Tracking in mmWave Radio Networks using Beam-Based DoD Measurements," in 8th International Conference on Localization and GNSS (ICL-GNSS), Jun. 2018, pp. 1-6.

[9] A. Shahmansoori, G. Seco-Granados, and H. Wymeersch, "Power Allocation for OFDM Wireless Network Localization Under Expectation and Robustness Constraints," IEEE Trans. Wireless Commun., vol. 16, no. 3, pp. 2027-2038, Mar. 2017. 
[10] G. Destino and H. Wymeersch, "On the Trade-off Between Positioning and Data-Rate for mm-Wave Communications," in IEEE International Conference on Communications Workshops (ICC Workshops), May 2017, pp. 797-802. 